\title{
Structural Superposition of Proteins With Unknown Alignment and Detection of Topological Similarity Using a Six-Dimensional Search Algorithm
}

\author{
Kay Diederichs \\ Fakultät für Biologie, Universität Konstanz, D-78434 Konstanz, Germany
}

\begin{abstract}
An algorithm for the rigidbody superposition of proteins is described and tested. No prior knowledge of equivalent residues is required. To find the common structural core of two proteins, an exhaustive grid search is conducted in three-dimensional angle space, and at each grid point a fast translation search in three-dimensional space is performed. The best superposition at given angle set is defined by that translation vector which maximizes the weighted number of equivalent $\mathrm{C} \alpha$ atoms. Filters using the information about the sequential character of the polypeptide chain are employed to identify that rotation and translation which yields the highest topological similarity of the two proteins. The algorithm is shown to find the best superposition of distantly related structures, and to be capable of finding similar structures to a given atomic model in the Brookhaven Protein Data Bank. In a search using granulocyte-macrophage colony-stimulating factor as a template, all other four-helix bundle cytokines with up-up-downdown topology were found to give the highest values of a topological similarity score, followed by interferon- $\beta$ and $-\gamma$ and those fourhelix bundles with the more common up-downup-down topology. In another example, the insertion domain of the long variant adenylate kinases is demonstrated to share its fold with rubredoxin. 잉 1995 Wiley-Liss, Inc.
\end{abstract}

Key words: classification of protein fold, protein evolution, spatial alignment algorithm, homology modeling, structural relationship, topology similarity score, common structural core, database searching

\section{INTRODUCTION}

The exponentially increasing number of macromolecular structures that evolve from X-ray crystallography and nuclear magnetic resonance spectroscopy makes it desirable to have algorithms that are capable of finding out structural relationships among atomic models. The recognition of topological similarities is important for model building by ho- mology, for the structural and evolutionary taxonomy of proteins and for the study of structure-function relationships. A spatial alignment is essential for the detailed structure-function comparison of molecular models, and molecular replacement studies can benefit from usage of different models that are in a common orientation and position in space.

A superposition of two structures given by their three-dimensional atomic coordinates $\{\mathbf{x}\}$ and $\{\mathbf{y}\}$ is usually based upon subsets of equivalent coordinates $\left(\left\{\mathbf{x}^{\mathbf{e}}\right\},\left\{\mathbf{y}^{\mathbf{e}}\right\}\right)$ and can be described by a rotation matrix $\mathbf{A}$ and translation vector $\mathbf{t}$ such that

$$
\sum_{\mathrm{i}}\left(\mathbf{A} \mathbf{x}_{i}^{\mathrm{e}_{i}}+\mathbf{t}-\mathbf{y}_{j(i)}^{\mathbf{e}}\right)^{2}=\text { Minimum }
$$

As any rotation in space can be generated with three angular variables, the problem of spatial superposition is six-dimensional.

Equation (1) is not well defined without specification of the subsets $\left\{\mathbf{x}^{\mathrm{e}}\right\},\left\{\mathbf{y}^{\mathrm{e}}\right\}$, and the set of correspondences $i \Leftrightarrow j(i)$. In the case of medium to high sequence homology, the subsets $\left\{\mathbf{x}^{\mathbf{e}}\right\},\left\{\mathbf{y}^{\mathbf{e}}\right\}$ of equivalent atoms are given by the homologous amino acids in a sequence alignment. However, for proteins many examples of similar three-dimensional structures without a significant degree of sequence homology are known. ${ }^{1-3}$ This means that

- the amount of similarity between two structures often cannot be predicted from the similarity of their sequences; instead, it has to be quantified using their superimposed three-dimensional, atomic coordinates;

- topologically equivalent positions in the polypeptide chain often cannot be determined from a sequence alignment; they have to be identi-

Abbreviations: AK, adenylate kinase; AK3, isoenzyme-3 of bovine mitochondrial matrix AK; SSE, secondary structure element; TSS, topological similarity score; FSS, fold similarity score; RSS, reverse-allowed similarity score; INSERT, insertion domain of the large variant AKs; CPU, central processing unit; GM-CSF, granulocyte-macrophage colony-stimulating factor; rms, root mean square.

Received March 14, 1995; revision accepted May 18, 1995.

Address reprint requests to Kay Diederichs, Fakultät für Biologie, Universität Konstanz, Postfach 5560, M656, D-78434 Konstanz, Germany. 
fied in a three-dimensional alignment of the structures if the sequence homology is low.

In the case of low or nondetectable sequence homology, efficient algorithms $\mathrm{s}^{4-7}$ for solving eq. (1) for $A$ and $t$ are often employed in the following four-step iterative algorithm:

1. visual inspection of the structures and identification of a few amino acids defining starting $\operatorname{sets}\left\{\mathbf{x}^{\mathbf{e}}\right\},\left\{\mathbf{y}^{\mathbf{e}}\right\}$

2. solve Eq. (1) for $\mathbf{A}$ and $\mathbf{t}$

3. identify a new set of atoms $\left\{\mathbf{x}^{\mathrm{e}}\right\},\left\{\mathbf{y}^{\mathrm{e}}\right\}$ satisfying $\left|\mathbf{A} \mathbf{x}_{i}{ }_{i}+\mathbf{t}-\mathbf{y}_{j(i)} \mathbf{e}_{j}\right|<d, d$ being a constant cutoff mostly chosen in the range 1.5 to $4.0 \AA$

4. go back to step 2 if the new sets $\left\{\mathbf{x}^{\mathrm{e}}\right\},\left\{\mathbf{y}^{\mathrm{e}}\right\}$ are different from the old sets.

Step 1 is time-consuming and subjective. Furthermore, except for the trivial case of only one matching atom, the procedure 1-4 is not guaranteed to converge to self-consistent sets $\left\{\mathbf{x}^{\mathrm{e}}\right\},\left\{\mathbf{y}^{\mathrm{e}}\right\}$ (for an example of oscillating behaviour, see PDB code 1RHG of Table IIa) and the global minimum of Eq. (1). Therefore, it is important to find good starting sets, and automatic methods for locating them have been devised. The process of selecting equivalent atoms in the presence of insertions and deletions can be guided by dynamic programming techniques, ${ }^{8}$ a method that is commonly used for sequence alignments. Several authors ${ }^{9-12}$ have applied this method to the problem of finding equivalent interatomic $\mathrm{C} \alpha-\mathrm{C} \alpha$ or $\mathrm{C} \beta-\mathrm{C} \beta$ distances in a distance matrix. Combinations with simulated annealing ${ }^{13}$ or a genetic algorithm, ${ }^{14}$ as well as a method derived from graph theory ${ }^{15}$ were also described. A Monte Carlo procedure was employed by Holm and Sander ${ }^{16}$ for optimizing a similarity score defined by $\mathrm{C} \alpha-\mathrm{C} \alpha$ distances. Most of these and other methods (for a review see Holm and Sander ${ }^{3}$ ) are complicated and depend on a number of parameters, for example, gap penalties for the alignment or schedules governing optimization protocols. Some of them require user intervention to achieve the best results.

An early six-dimensional search method ${ }^{17}$ was computationally slow and could therefore only be performed on a coarse grid, thus potentially missing the best orientation. In this paper a general and fully automatic six-dimensional grid search method, which finds the global maximum of a scoring function in a computationally efficient manner, is described. Due to the conceptual simplicity of the method, no parameters that require modification for particular cases are involved. Several scoring functions are discussed and examples for the application of the algorithm are given.

\section{METHODS}

A six-dimensional search on a computer using a suitable target function is the conceptually simplest approach to the problem of superimposing two threedimensional entities. It resembles the human action of rotating and translating two wire-frame models in space until they seem to match best. At first sight, the resources required for a six-dimensional search on a grid of suitable spacing may seem prohibitive, even though the computational capabilities of contemporary workstations are higher than those of supercomputers a decade ago. However, a very fast algorithm for the calculation of the translation function is described below.

In the following, a simplification of the superposition problem is achieved by accepting only $\mathrm{C} \alpha$ atoms as members of the sets $\{\mathbf{x}\}$ and $\{\mathbf{y}\}$, in effect reducing the number of atoms to be considered by about a factor of eight.

A rigid-body superposition algorithm works best if domains of proteins are considered. Neglecting their internal plasticity, these can be described as rigid bodies in a first approximation. Thus, if one suspects large domain movements (e.g., see Schulz et al. ${ }^{18}$ ), the domains of two multidomain proteins should be superimposed separately.

\section{Parameterization of Angle Space}

If a comparison is done between two structures without any internal symmetry, an Eulerian angle $\left(\Theta_{1}, \Theta_{2}, \Theta_{3}\right)$ range with $0 \leq \Theta_{1}<360^{\circ}, 0 \leq \theta_{2} \leq$ $180^{\circ}, 0 \leq \theta_{3}<360^{\circ}$ has to be considered. In the algorithm described here, the number of unique orientations required to cover the same range of orientations is reduced by a factor of about $(2 / \pi)$ by using the pseudo-orthogonal angles $\left(\theta_{+}, \Theta_{2}, \theta_{-}\right)$introduced by Lattman. ${ }^{19}$ The Lattman angle space is given by $\left(0 \leq \theta_{+}<720^{\circ}, 0 \leq \theta_{2} \leq 180^{\circ}, 0 \leq \theta_{-} \leq\right.$ $\left.360^{\circ}\right)$ and the step size in $\Theta_{+}$and $\theta_{-}$is $\Delta \theta_{2} /$ $\cos \left(\Delta \Theta_{2} / 2\right)$ and $\Delta \Theta_{2} / \sin \left(\Delta \Theta_{2} / 2\right)$, respectively. For example, if a step size of $\Delta \theta_{2}=10^{\circ}$ is used, 22104 unique orientations in Eulerian angle space result. The use of Lattman angles reduces this number to 15,589 . Contrary to Eulerian angles, Lattman angles associate equal volumes of angle space with each sample point, with unbiased estimates of statistical parameters (like average and standard deviation) of the function values evaluated on the sampling grid.

\section{Calculation of the Fast Translation Function}

To identify the correct superposition, a three-dimensional translation search has to be performed in cartesian coordinate space for each of the unique orientations. As a first step, it is convenient to subtract the respective centers of mass from all coordinates of the sets $\{\mathbf{x}\}$ and $\{\mathbf{y}\}$.

The simplest way to calculate a translation function $Q_{\mathbf{A}}(t)$ is done by shifting the rotated coordinate set $\{\mathbf{A} \mathbf{x}\}$ by a vector $t$ and to count the number $Q_{\mathbf{A}}$ of coordinates $\mathbf{A x}_{i}+\mathbf{t}$ that are within a certain distance $d$ from any atom $\mathbf{y}_{j}$. This can be done for all 
values of $\mathbf{t}$ on a three-dimensional grid that are shorter than $D$, the maximum expected shift distance. The maximum of $Q_{\mathrm{A}}$ then yields the best translation $t_{b}$. The drawback of this straightforward algorithm is that at each grid point $\mathbf{t}$ it requires a computational effort proportional to the product of the number of atoms in the sets $\{\mathbf{x}\}$ and $\{\mathbf{y}\}$.

A much faster implementation of the translation function for the sets $\{\mathbf{x}\}$ and $\{\mathbf{y}\}$ is possible by using a two-step procedure. As a first step, the three-dimensional array $q_{\mathbf{A}}(\mathbf{t})$ is calculated:

$$
q_{\mathbf{A}}(\mathbf{t})=\sum_{i} \sum_{j} w_{i j} \delta\left(\mathbf{t}-\left[\mathbf{y}_{j}-\mathbf{A} \mathbf{x}_{i}\right]\right)
$$

where

$$
\delta(\mathbf{u}-\mathbf{v})= \begin{cases}1 & \text { if } u=v \\ 0 & \text { otherwise }\end{cases}
$$

$\left[\mathbf{y}_{j}-\mathbf{A x}_{i}\right]$ denotes the gridpoint that is nearest to $\mathbf{y}_{j}-\mathbf{A} \mathbf{x}_{i}$, and

$w_{i j}=\left\{\begin{array}{l}1 \text { if only structural information should be considered } \\ \text { or } \\ w_{i j} \text { element of an amino acid exchange matrix }\end{array}\right.$ $w_{i j}$ is a weight ${ }^{20}$ defined by the types of the amino acids at positions $i, j$. It denotes the probability of an exchange of the amino acid type a position $i$ to the type of residue at position $j$. If one wants to detect similarities based only on spatial position information, $w_{i j}$ would be chosen as being independent of the types of amino acids $i, j$. However, if an evolutionary relationship between the two proteins can be anticipated, $w_{i j}$ can be used to increase the contribution of pairs $\{i, j\}$ with similar or the same amino acid types and to downweight, for example, a superposition of a hydrophobic onto a hydrophilic amino acid.

In Eq. (2), $q_{\mathrm{A}}(\mathrm{t})$ counts the number of all those parallel vectors $\left(\mathbf{y}_{j}-\mathbf{A x} \mathbf{x}_{i}\right)$ of equal length leading to a common translation $t$. In practice, it suffices to calculate $q_{\mathbf{A}}(\mathbf{t})$ on a $1 \AA$ grid. Algorithmically, $q_{\mathbf{A}}(\mathbf{t})$ can therefore be evaluated as a double loop running over all pairs $\left\{\mathbf{x}_{i}, \mathbf{y}_{j}\right\}$. Inside the loop, the value of $q_{\mathbf{A}}$ at the grid point $\mathbf{t}$ nearest to $\left(\mathbf{y}_{j}-\mathbf{A x}_{i}\right)$ is incremented by $w_{i j}$ : This is only done if the length of $\mathbf{t}$ is less or equal to $D$, the largest expected shift distance. $Q_{A}(t)$ is found in a second step: the array $q_{A}(t)$ is scanned and $Q_{\mathbf{A}}(t)$ is assigned the sum of the $q_{\mathrm{A}}\left(\mathrm{t}^{\prime}\right)$ values of the grid points in a $3 \times 3 \times 3$ box centered on $t$. Thus, in this algorithm the distance parameter $d$ of the previous algorithm corresponds to accepting difference vectors from adjacent gridpoints. If a $1 \AA$ grid is used, difference vectors $t^{\prime}$ in a distance $d$ between 1.5 and $1.5,3$ from $t$ contribute to $Q_{\mathbf{A}}(\mathbf{t})$. The highest value of $Q_{\mathbf{A}}$ obtained after scanning the whole array is considered to be the result $Q_{\mathbf{A}}\left(\mathbf{t}_{b}\right)$ of the translation function at rotation $\mathbf{A}$, together with the translation $t_{b}$ at which it is found.

In this fast version of the translation function, the double loop over all pairs $\left\{\mathbf{x}_{i}, \mathbf{y}_{j}\right\}$ is calculated only once, instead of calculating it at every grid point $t$. The only computation that has to be performed at every grid point is the summation of $q_{\mathrm{A}}(\mathrm{t})$ over 27 gridpoints. By using the fourier transform of $q_{\mathrm{A}}(\mathrm{t})$, this summation could even be replaced with a single multiplication.

It should be noted that the algorithm can be efficiently implemented on parallel computers or clusters of workstations computing in parallel because the results of the translation function at different rotation angles do not depend upon each other.

\section{Filtering the Results of the Fast Translation Function}

For the computation of $Q_{\mathbf{A}}\left(t_{b}\right)$, no use has been made of the sequential nature of the coordinates. This means, that up to the filtering stage discussed below the atomic coordinates may be unsorted: any three-dimensional assembly of points could be evaluated against any other assembly of points. Although the $Q_{\mathrm{A}}$ score reliably identifies the translation $t_{b}$ which maximizes the number of equivalent $\mathrm{C} \alpha$ atoms for a particular rotation matrix $\mathrm{A}$, the maximum of $Q_{\mathbf{A}}$ over all $A\left\{\max _{\mathbf{A}}\left[Q_{\mathbf{A}}\left(\mathbf{t}_{b}\right)\right]\right\}$ not always corresponds to that rotation which is most meaningful in the sense of topological or folding similarity. The purpose of filtering is therefore the evaluation of all $A / t_{b}$ combinations in terms of the requirement that superimposed atoms should occur in sequential stretches in the two proteins.

For a given result $t_{b}$ of a translation function at a given angle combination $\mathbf{A}$, the amount of local or global sequentiality may be evaluated in a filtering routine. The result of the filter provides further information concerning the usefulness of this particular rotation/translation combination. "Local sequentiality" means the following: if $\mathbf{x}_{i}$ is within a certain distance $d$ of $\mathbf{y}_{j}$, then $\mathbf{x}_{i}+{ }_{1}$ should be within $d$ of $\mathbf{y}_{j+1}$ or $\mathbf{y}_{j-1}$. The number of forward sequential matches $\left(\mathbf{x}_{i}+1\right.$ within $d$ of $\left.\mathbf{y}_{j}+1\right)$ is counted by $\operatorname{LSF}_{\mathbf{A}}\left(\mathbf{t}_{b}\right)$, the number of reverse sequential matches $\left(\mathbf{x}_{i}+1\right.$ within $d$ of $\left.\mathbf{y}_{j-1}\right)$ by $\operatorname{LSR}_{\mathbf{A}}\left(\mathbf{t}_{b}\right)$. The numbers of atoms $\operatorname{LSF}_{\mathbf{A}}\left(t_{b}\right), \operatorname{LSR}_{\mathbf{A}}\left(t_{b}\right)$ involved in locally sequential stretches give an important hint about the quality of the alignment. For example, only these atoms which are counted in $\operatorname{LSF}_{\mathbf{A}}\left(\mathbf{t}_{b}\right)$ should usually be used in a least-squares improvement of superposition, as any other matches represent spurious equivalences.

"Global sequentiality" GS $_{\mathbf{A}}\left(\mathrm{t}_{\boldsymbol{b}}\right)$ can be measured by the correlation coefficient between the indices $i, j$ of matching atom pairs. Only those atoms that contribute to $\operatorname{LSF}_{\mathbf{A}}\left(\mathbf{t}_{b}\right)$ are used in calculating $\mathrm{GS}_{\mathbf{A}}\left(\mathrm{t}_{\boldsymbol{b}}\right)$. This quantity is relatively insensitive with respect to insertions and deletions. It is high if the order and direction of matching structural elements are the same in both structures. As the correlation coeffi- 
cient is a number between -1 and 1 , it is a normalized measure, not depending on the absolute lengths of the sequences.

The cost of computing the filter values $\operatorname{LSF}_{\mathbf{A}}\left(\mathbf{t}_{b}\right)$, $\operatorname{LSR}_{\mathbf{A}}\left(\mathbf{t}_{b}\right)$ and $\mathrm{GS}_{\mathbf{A}}\left(\mathbf{t}_{b}\right)$ is low, as they are only evaluated once for each rotation matrix $\mathbf{A}$, at the translation $\mathbf{t}_{b}$ corresponding to the maximum of $Q_{\mathrm{A}}$.

\section{Scoring Functions}

Three different yet interesting ways of how two structures may be similar can be postulated:

1. Topological similarity, preserving order and direction of $\alpha$-helical or $\beta$-strand secondary structure elements (SSEs);

2. similarity of fold: superimposable SSEs with same direction are present but the structures differ in the connections between these SSEs; different connections result in different sequential order of SSEs; and

3. same as (2), but additionally allowing for different directions of SSEs.

In order to combine both local and global similarity filters into one measure, a topological similarity score is defined as

$$
\text { TSS }=\max _{\mathbf{A}}\left[\operatorname{LSF}_{\mathbf{A}}\left(t_{b}\right) \times \operatorname{GS}_{\mathbf{A}}\left(t_{\mathbf{b}}\right)\right]
$$

For measuring topological similarity, $\mathrm{GS}_{\mathbf{A}}\left(\mathbf{t}_{b}\right)$ or LS$F_{A}\left(t_{b}\right)$ alone are not suitable: a low number of matched amino acid residues might yield a high, yet insignificant correlation, and a high $\operatorname{LSF}_{\mathbf{A}}\left(\mathbf{t}_{b}\right)$ alone does not ensure the same order of the elements of secondary structure in both structures. The product of $\operatorname{LSF}_{\mathbf{A}}\left(\mathbf{t}_{b}\right)$ and $\mathrm{GS}_{\mathbf{A}}\left(\mathbf{t}_{b}\right)$ yields a high TSS if two structures show the same sequential order and direction of secondary structures, i.e., case 1 above.

Using FSS $=\max _{\mathbf{A}}\left[\operatorname{LSF}_{\mathbf{A}}\left(\mathbf{t}_{b}\right)\right]$ as a fold similarity score is suitable for the detection of structures with similar fold, but possibly different topology (case 2 above).

A useful definition for a reverse-allowed similarity score is RSS $=\max _{\mathbf{A}}\left[\operatorname{LSF}_{\mathbf{A}}\left(\mathbf{t}_{b}\right)+\operatorname{LSR}_{\mathbf{A}}\left(\mathbf{t}_{b}\right)\right]$, allowing detection of those similar folds that include direction reversals of SSEs (case 3 above).

\section{Test Data}

For the evaluation of the algorithm, the structures of granulocyte-macrophage colony-stimulating factor ${ }^{21}$ (GM-CSF) (PDB code 1GMF, 119 amino acid residues), the $\mathrm{C}$-terminal membrane-insertion domain of colicin $\mathrm{A}^{22}$ (PDB code 1COL, 192 amino acid residues) and residues 125 to 159 of bovine mitochondrial matrix adenylate kinase ${ }^{23}$ (AK3, PDB code 1AK3) were each compared to a subset of structures $^{24,25}$ of the Protein Data Bank ${ }^{26}$ (PDB). The subset (version of October 1994) consists of those entries of the PDB which show less than $90 \% \mathrm{mu}-$ tual sequence homology and spans the whole space of presently known folds. The 612 atomic structures used for this study are the result of X-ray crystallo- graphic analysis. In those cases where noncrystallographic symmetry generated more than one copy of the molecule in the asymmetric unit, only the first of these was used.

GM-CSF is a member of the family of four-helix bundle cytokines with the otherwise unobserved upup-down-down topology. Also present in the 612structure subset are interleukin-2 (PDB code 3INK, 121 amino acid residues), interleukin-4 (1RCB, 129 amino acid residues), human growth hormone (1HUW/3HHR, 166/184 amino acid residues), macrophage colony-stimulating factor (1HMC, 148 amino acid residues), and granulocyte colony-stimulating factor (1RHG/1BGC/1BGE, 145/158/159 amino acid residues). These cytokines were reported $^{27}$ to be similar to interferon- $\beta$ and interferon- $\gamma$, which are topologically related ${ }^{28}$ and are also present in the subset (1IFA; 1HIG/2RIG/1RFB). Two other members of this superfamily, leukemia inhibitory factor ${ }^{29}$ and interleukin $-5^{30}$ are not present in the Protein Data Bank.

The membrane-insertion domain of colicin A topologically resembles the globins and phycocyanin as was noted earlier. ${ }^{2,31}$ Phycocyanin (1CPC, 162 amino acid residues) and several globins are present in the subset. The structural similarity of colicin A and the globins is not paralleled by functional relationship or sequence homology, and evolution converging to the same structure can be assumed. The detection of topological similarity between these proteins was the result of an automatic data bank search with the program DALI. ${ }^{16}$ These structures differ significantly in the number and relative orientation of helices such that their similarities evaded detection by the human eye. The databank search with colicin $A$ is therefore a stringent test case for any structural superposition algorithm.

The long variants of the adenylate kinases (AKs) differ from the short ones by an insertion of 27 residues after helix 6 (out of 9) of the core domain common to all AKs. These and some adjacent residues form a domain which is built up by two sandwiched $\beta$-meanders. During catalysis, this domain (INSERT), and another domain which binds adenosine monophosphate, seem to rotate as rigid bodies with respect to the core domain. Relative orientations observed in crystal structures representing different stages of the catalytic cycle suggest a possible rotation range of INSERT by up to about $90^{\circ} .{ }^{18}$ The sequence and the structure of INSERT are well conserved among the long variants of the AKs. To the best of the author's knowledge, a similarity of the fold of this domain to any other known protein structure of a different family has not yet been described in the literature.

Neither GM-CSF, nor colicin A, nor the INSERT domain of the AKs shows significant sequence homology to any of those proteins with which they share the fold. 
TABLE I. Weights $w_{i j}$ Used in the Test Cases

\begin{tabular}{lccc}
\hline$i / j$ & Hydrophilic* & Hydrophobic & Aromatic $^{\ddagger}$ \\
\hline Hydrophilic* $^{*}$ & 1.0 & 0.3 & 0.6 \\
Hydrophobic $^{\dagger}$ & 0.3 & 1.0 & 0.6 \\
Aromatic $^{\ddagger}$ & 0.6 & 0.6 & 1.0 \\
\hline
\end{tabular}

${ }^{*}$ Hydrophilic residues: Arg, Asp, Asn, Glu, Gln, Lys, Ser, Thr ${ }^{\dagger}$ Hydrophobic residues: Ala, Cys, Gly, Ile, Leu, Met, Pro, Val. ${ }^{\ddagger}$ Aromatic residues: Tyr, Phe, Trp, His.

\section{The Program}

The program was written in Fortran-77 and tested under the VAX/VMS and several UNIX operating systems. Input to the program is two coordinate sets in PDB format, the first serving as a reference. The output is the second coordinate set, rotated and translated with those parameters corresponding to the highest value of a scoring function (see below). A final report about the best solution found for each possible scoring function $\left[\max _{\mathbf{A}}\left(Q_{\mathbf{A}}\left(t_{b}\right)\right]\right.$, TSS, FSS, and RSS) is printed. All calculations are based upon $\mathrm{C} \alpha$ atoms only.

The search is performed in steps of $10^{\circ}$, and a local $5^{\circ}$ search is performed additionally if one of the scoring functions is larger than a threshold. A $10^{\circ}$ grid spacing ensures a maximum rotation angle error of $5,3^{\circ}$. For a domain of typical size (radius $12 \AA$ ), the coordinate error of the outermost atoms amounts to less than half of the C $\alpha$ separation, allowing a correct match. In the case of even larger domains, the contribution of the atoms in the inner $12 \AA$ shell still produces a significant peak in the scoring functions.

The computational result of a six-dimensional superposition run between two proteins is a list of the values of $t_{b}, Q_{A}\left(t_{b}\right)$, the number of matching residues and the filter values $\operatorname{LSF}_{\mathbf{A}}\left(t_{b}\right), \operatorname{LSR}_{\mathbf{A}}\left(t_{b}\right)$, and $\mathrm{GS}_{\mathbf{A}}\left(\mathrm{t}_{\boldsymbol{b}}\right)$ for each angle set $\mathbf{A}$. The list is written to a file to enable later detection and evaluation of suboptimal solutions.

The weights $w_{i j}$ used for the test cases are given in Table I. For the calculation of the filter functions, the distance cutoff $d$ employed for assigning equivalence of atom pairs was $2.5 \AA$.

The average CPU time for a superposition of two medium-sized proteins is about $6 \mathrm{~min}$ on a Silicon Graphics workstation with R4000 CPU. The program can be obtained from the author upon request.

\section{RESULTS AND DISCUSSION}

For the present work, the main focus was on the detection of topological similarity. Therefore, the subset PDB entries are sorted on their TSS in Table IIa-c. The table lists those subset entries with a TSS higher than the mean of TSS (Table IIa, 22.41; Table IIb, 25.80; Table IIc, 15.73) plus twice the standard deviation $(6.54,6.96$, and 2.86 , respectively) of the TSS scores.

The four-step least-squares algorithm as imple- mented in "O"32, using a $2.5 \AA$ cutoff, requiring at least two subsequent matching residues and starting with the alignment found by the search program, was iterated until convergence. The root mean square (rms) agreement of matching $\mathrm{C} \alpha$ positions as found by the search program and as improved in " $O$ " are tabulated, together with the respective number of matching atoms.

Table IIa shows the results using GM-CSF as the search template for the other 611 structures of the subset. Here, all those proteins which are known to have the same fold as GM-CSF (interleukin-2 and -4 , G-CSF, M-CSF, and human growth hormone) yield the highest TSSs found by the program. It is remarkable that the lengths of the sequences that share the same fold vary between 121 and 184 amino acid residues. Following these proteins with up-up-down-down topology, interferon- $\beta$ and several four-helix bundles with the conventional updown-up-down topology (myohemerythrin, 2MHR; hemerythrin, 2HMZ; cytochrome $c^{\prime}, 1 \mathrm{BBH}$ and 2CCY; cytochrome $b_{562}, 256 \mathrm{~B}$ ) are found. The high degree of topological similarity between GM-CSF and the cytokines reveals itself by high values of GS $(\geq 0.98$ ). Lower values of GS $(0.80-0.92)$ reflect the different topology of the up-down-up-down four-helix bundles. A high value of GS (1.0) is also found for interferon- $\gamma$, a molecule consisting of six $\alpha$-helices. In this case, helices 1 to 3 of GM-CSF are superimposed with helices $A, C$, and D of interferon- $\gamma$; helix B playing the role of the long "overhand" connection between helices 1 and 2 of GM-CSF. For interferon- $\beta$, helices 1 to 4 of GM-CSF match helices A, C, $\mathrm{D}$, and $\mathrm{F}$, the crossing angle between $\mathrm{C}$ and 2 amounting to about $30^{\circ}$. As in interferon- $\gamma$, the loop called $\mathrm{B}^{*}$ (nomenclature of Ealick et al. ${ }^{28}$ ) and helix $F$ play the roles of the long loops in GM-CSF. The sequence alignments implied by the structural superpositions are in good agreement with those of Rozwarski et al. ${ }^{33}$ Substructures, in which some helices match those of GM-CSF, are found by the program in the much larger atomic models of ligninperoxidase (343 residues) and glucoamylase (471 residues).

Results of the search with colicin A as a template are given in Table IIb. Here, phycocyanin (1CPC) and several hemoglobins (1HLB, 1ITH, 1ECO, 1YMC) score higher than three standard deviations above the average TSS, followed by all other globins in the subset and several helix-bundle or helix-containing structures which match mainly because two or more of their helices can be superimposed with those of colicin A. The TSS of phycocyanin is nearly five standard deviations above the mean, indicating a highly significant agreement of topologies. The sequence alignment obtained by structural superposition of the globins and colicin $A$ is in register with that obtained by Holm and Sander. ${ }^{2}$

The search with the INSERT domain of AK3 (Ta- 
TABLE II. Searches With GM-CSF, Colicin A, and Residues 125-159 of AK3 as Templates Against a Subset of the Protein Data Bank.

\begin{tabular}{|c|c|c|c|c|c|c|c|}
\hline Compound & PDB code & $N_{\text {residues }}$ & $N_{\text {match }}^{\dagger}$ & $\mathrm{LSF}^{\ddagger}$ & $\mathrm{GS}^{\S}$ & $\operatorname{RMSD}^{* *}(\AA)$ & $\begin{array}{c}\text { TSS }= \\
\mathrm{LSF} \times \text { GS }\end{array}$ \\
\hline \multicolumn{8}{|c|}{ a. GM-CSF as a Template } \\
\hline Interleukin-4 & $1 \mathrm{RCB}$ & 129 & 71 & 63 & 0.98 & $1.53 / 1.37(61)$ & 61.74 \\
\hline G-CSF (human) & 1RHG & 145 & 73 & 59 & 0.98 & $1.62 / *$ & 57.82 \\
\hline G-CSF (bovine) & 1BGC & 158 & 78 & 57 & 1.00 & $1.64 / 1.59(57)$ & 57.00 \\
\hline M-CSF & $1 \mathrm{HMC}$ & 148 & 69 & 55 & 1.00 & $1.69 / 1.62(49)$ & 55.00 \\
\hline Interleukin-2 & 3INK & 121 & 65 & 55 & 0.99 & $1.48 / 1.38(57)$ & 54.45 \\
\hline G-CSF (canine) & 1BGE & 159 & 75 & 55 & 0.99 & $1.77 / 1.50(49)$ & 54.45 \\
\hline Human growth hormone w/ receptor & $3 \mathrm{HHR}$ & 184 & 72 & 49 & 1.00 & $1.65 / 1.58(51)$ & 49.00 \\
\hline Human growth hormone & 1HUW & 166 & 69 & 48 & 0.98 & $1.53 / 1.40(44)$ & 47.04 \\
\hline Interferon- $\beta$ & IIFA & 159 & 73 & 47 & 0.99 & $1.68 / 1.29(41)$ & 46.53 \\
\hline Myohemerythrin & $2 \mathrm{MHR}$ & 118 & 63 & 47 & 0.92 & $1.69 / 1.53(46)$ & 43.24 \\
\hline Hemerythrin & $2 \mathrm{HMZ}$ & 114 & 59 & 45 & 0.88 & $1.63 / 1.71(40)$ & 39.60 \\
\hline Cytochrome $c^{\prime}$ (chromatium vinosum) & $1 \mathrm{BBH}$ & 131 & 59 & 45 & 0.88 & $1.62 / 1.57(39)$ & 39.60 \\
\hline Lignin-peroxidase & $1 L G A$ & 343 & 55 & 43 & 0.92 & $1.54 / 1.28(27)$ & 39.56 \\
\hline Colicin A & $1 \mathrm{COL}$ & 197 & 64 & 40 & 0.96 & $1.53 / 1.38(37)$ & 38.40 \\
\hline Interferon- $\gamma$ (rabbit) & 2RIG & 119 & 45 & 38 & 1.00 & $1.66 / 1.44(36)$ & 38.00 \\
\hline Glucoamylase & 1DOG & 471 & 68 & 40 & 0.94 & $1.36 / 0.77(31)$ & 37.60 \\
\hline Cytochrome $b_{562}$ & $256 \mathrm{~B}$ & 106 & 58 & 40 & 0.92 & $1.54 / 1.50(40)$ & 36.80 \\
\hline Interferon- $\gamma$ (human) & 1HIG & 123 & 41 & 36 & 1.00 & $1.51 / 1.14(35)$ & 36.00 \\
\hline \multicolumn{8}{|c|}{ b. Colicin A as a Template } \\
\hline C-Phycocyanin & $1 \mathrm{CPC}$ & 162 & 81 & 60 & 1.00 & $1.46 / 1.42(57)$ & 60.00 \\
\hline Hemoglobin (innkeeper worm) & IITH & 141 & 79 & 53 & 0.99 & $1.78 / 1.45(45)$ & 52.47 \\
\hline Hemoglobin (sea cucumber) & 1HLB & 157 & 81 & 53 & 0.99 & $1.69 / 1.45(40)$ & 52.47 \\
\hline Hemoglobin (erythrocruorin) & $1 \mathrm{ECO}$ & 136 & 79 & 51 & 1.00 & $1.66 / 1.48(34)$ & 51.00 \\
\hline Hemoglobin (horse heart) & $1 Y M C$ & 153 & 86 & 48 & 1.00 & $1.66 / 1.29(39)$ & 48.00 \\
\hline Hemoglobin (yellow lupin) & $2 \mathrm{LH} 2$ & 153 & 75 & 45 & 1.00 & $1.75 / 1.46(47)$ & 45.00 \\
\hline Hemoglobin (human) & $1 \mathrm{BAB}$ & 142 & 78 & 45 & 1.00 & $1.64 / 1.49(41)$ & 45.00 \\
\hline Cytochrome $c^{\prime}$ & $1 \mathrm{BBH}$ & 131 & 59 & 45 & 0.96 & $1.55 / 1.39(39)$ & 43.20 \\
\hline Hemoglobin (ark clam) & $3 \mathrm{SDH}$ & 145 & 79 & 43 & 1.00 & $1.70 / 1.24(33)$ & 43.00 \\
\hline Hemoglobin (sea hare) & 2FAL & 145 & 70 & 43 & 1.00 & $1.53 / 1.20(31)$ & 43.00 \\
\hline Myoglobin (seal) & 1MBS & 153 & 66 & 43 & 1.00 & $1.70 / 1.29(35)$ & 43.00 \\
\hline Hemoglobin (lucina pectinata) & 1FLP & 142 & 64 & 43 & 0.99 & $1.54 / 1.47(34)$ & 42.57 \\
\hline Hemerythrin & $2 \mathrm{HMZ}$ & 114 & 62 & 44 & 0.96 & $1.32 / 1.24(44)$ & 42.24 \\
\hline Hemoglobin (marine bloodworm) & $2 \mathrm{HBG}$ & 147 & 71 & 42 & 1.00 & $1.65 / 1.36(34)$ & 42.00 \\
\hline Myoglobin (tuna) & $1 \mathrm{MYT}$ & 146 & 75 & 42 & 1.00 & $1.64 / 1.51(39)$ & 42.00 \\
\hline Hemoglobin (horse) & $2 \mathrm{MHB}$ & 141 & 71 & 42 & 0.99 & $1.75 / 1.64(25)$ & 41.58 \\
\hline Hemoglobin (sea lamprey) & 2LHB & 150 & 75 & 42 & 0.99 & $1.63 / 1.60(46)$ & 41.58 \\
\hline Protein R2 of ribonucleotide reductase & 1RIB & 340 & 91 & 48 & 0.86 & $1.59 / 1.26(35)$ & 41.28 \\
\hline Apolipophorin & 1AEP & 153 & 65 & 42 & 0.98 & $1.51 / 1.11(36)$ & 41.16 \\
\hline Hemoglobin (deer) & $1 \mathrm{HDS}$ & 141 & 68 & 45 & 0.91 & $1.68 / 1.38(37)$ & 40.95 \\
\hline Myoglobin (sperm whale) & 2MGE & 154 & 75 & 48 & 0.84 & $1.74 / 1.47(35)$ & 40.32 \\
\hline Hemoglobin (pig) & 2PGH & 141 & 75 & 40 & 1.00 & $1.64 / 1.47(34)$ & 40.00 \\
\hline Cytochrome $b_{562}$ & $256 \mathrm{~B}$ & 106 & 54 & 40 & 1.00 & $1.33 / 0.71(34)$ & 40.00 \\
\hline Hemoglobin (bovine) & $1 \mathrm{HDA}$ & 141 & 72 & 40 & 1.00 & $1.54 / 1.35(39)$ & 40.00 \\
\hline \multicolumn{8}{|c|}{ c. Residues 125-159 of AK3 as a Template } \\
\hline Adenylate kinase $(E$. coli) & 1AKE & 214 & 35 & 35 & 1.00 & $1.16 / 0.88(35)$ & 35.00 \\
\hline Rubredoxin (Clostr. pasteur.) & $5 \mathrm{RXN}$ & 54 & 27 & 27 & 0.98 & $1.74 / 1.56(25)$ & 26.46 \\
\hline Rubredoxin (Desulf. gigas) & 1RDG & 52 & 26 & 26 & 0.98 & $1.71 / 1.59(25)$ & 25.48 \\
\hline Rubredoxin (Clostr. pasteur.) & $8 \mathrm{RXN}$ & 52 & 26 & 25 & 0.98 & $1.72 / 1.60(26)$ & 24.50 \\
\hline Rubredoxin (Pyroc. furiosus) & 1CAA & 53 & 26 & 25 & 0.98 & $1.57 / 1.48(24)$ & 24.50 \\
\hline Rubredoxin (Desulf. desulf.) & $6 \mathrm{RXN}$ & 45 & 25 & 24 & 1.00 & $1.70 / 1.42(22)$ & 24.00 \\
\hline Retinol-binding protein & $10 P B$ & 133 & 24 & 23 & 0.98 & $1.78 / 1.07(17)$ & 22.54 \\
\hline Met aminopeptidase & 1MAT & 263 & 28 & 23 & 0.96 & $1.68 / 1.40(13)$ & 22.08 \\
\hline Kallikrein A & 2PKA & 80 & 25 & 22 & 1.00 & $1.83 / 1.34(18)$ & 22.00 \\
\hline Antigen-binding fragment ( $\mathrm{Fab}$ ) & 1MAM & 214 & 26 & 22 & 0.99 & $1.60 / 1.46(21)$ & 21.78 \\
\hline Rat mast cell protease & 3RP2 & 217 & 28 & 25 & 0.87 & $1.59 / 1.39(20)$ & 21.75 \\
\hline Trypsin w/ benzamidine inhibitor & 2TBS & 220 & 27 & 25 & 0.87 & $1.54 / 1.22(18)$ & 21.75 \\
\hline Proteinase B & 3SGB & 185 & 27 & 23 & 0.94 & $1.47 / 1.24(20)$ & 21.62 \\
\hline
\end{tabular}

*Four-step iterative least-squares algorithm oscillates between 1.26 (38) and 1.31 (42).

${ }^{\dagger} N_{\text {match }}$, number of C $\alpha$ atoms at rotation/translation corresponding to best TSS which are within a $2.5 \AA$ distance of any C $\alpha$ atom of the other molecule.

${ }^{\ddagger} \mathrm{LSF}$, number of $\mathrm{C} \alpha$ atoms in a row of at least two residues at rotation/translation corresponding to best TSS. Those atoms that count for $N_{\text {match }}$ are considered.

${ }^{s} \mathrm{GS}$, correlation of sequence numbers at rotation/translation corresponding to best TSS. Those residues that count for LSF are considered.

**RMSD, rms deviation at rotation/translation corresponding to best TSS of those C $\alpha$ atoms that count for LSF / rms deviation after application of the 4-step iterative least-squares algorithm (corresponding number of contributing $\mathrm{C} \alpha$ atoms in parentheses). 

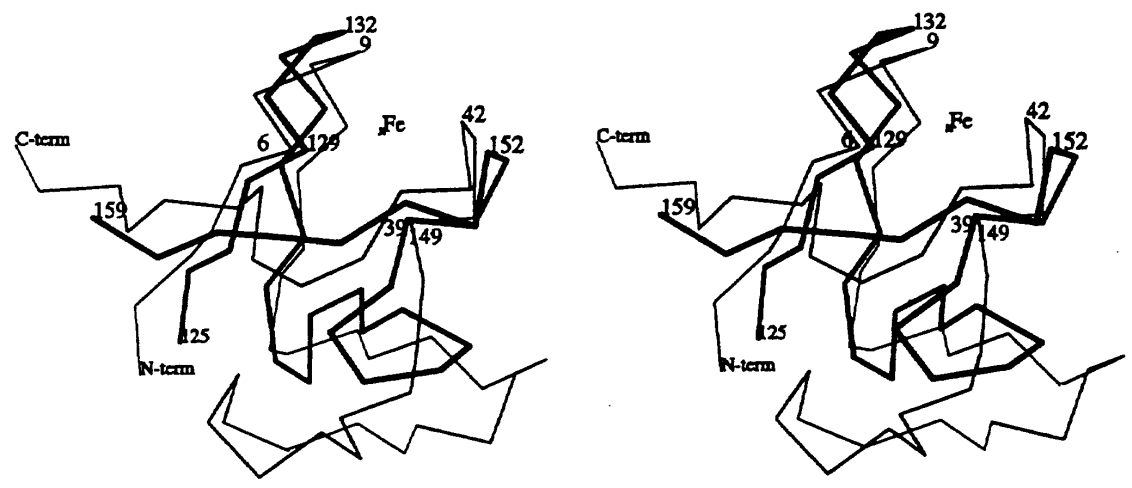

Fig. 1. Stereo view (produced with $\mathrm{O}^{32}$ ) of superposition ( $\mathrm{C}_{\alpha}$ traces) of residues 125-159 (INSERT) of AK3 (heavy lines) and rubredoxin (5RXN, thin lines). Residues Cys-6, Cys-9, Cys-39, and Cys-42 of rubredoxin, which topologically match the zinc-binding Cys residues conserved in most Gram-positive AKs (residue numbers in 1AK3: 129, 132, 149, 152), coordinate an Fe atom.

ble IIc) scored highest for the equivalent domain of AK from $E$. coli, as was expected from their high structural homology. All five rubredoxin structures present in the subset receive the next highest TSS. For these, the residues matched by the algorithm form a significant part of the whole protein (Fig. 1). In principle, it seems plausible that a convergent evolutionary process might have produced a very similar, stable fold of a small domain consisting of short antiparallel $\beta$-meanders in both cases. However, there is evidence that the topological agreement does not occur by chance, nor in a functionally unrelated fashion: the superposition generates a structural alignment of the $\mathrm{Fe}$-ion coordinating Cys-6, Cys-9, Cys-39, and Cys-42 of rubredoxin with residues His-129, Ser-132, Asp-149, and Thr-152 of AK3.

Notably, the homologous positions in B. stearothermophilus $\mathrm{AK}$ are substituted by cysteines which were demonstrated ${ }^{34,35}$ to bind a structural zinc atom. Thus, both Gram-positive bacterial AKs and rubredoxin bind a metal ion in a topologically equivalent place. As there is no known functional role of the zinc atom, and the short variant AKs completely lack the INSERT domain, one could imagine the insertion of a gene coding for an ancestral metal-binding domain into the precursor gene of today's large variant AKs, a gene whose divergent evolution on the other hand produced the contemporary rubredoxins.

In two of the test cases, topologically similar structures were known from previous studies. These structures were indeed found by the algorithm, and received the highest TSS score in the database search. The ordering produced by sorting on TSS matches well the degree of similarity perceived by visual inspection. In both the cytokine and the globin family, all family members are found, with more remotely related structures receiving a lower TSS, as in the case of the interferons. On the con- trary, the rms deviation of $\mathrm{C} \alpha$ positions is not a direct function of the degree of topological similarity.

These results attest to the performance of the algorithm, which produces information about distant structural similarities between the search templates and the members of a subset of the Protein Data Bank. In these and other database searches (data not shown) the algorithm has proved to be both accurate in the superpositions produced and sensitive to any topological or fold similarity. The potential to find hitherto unknown though interesting similarities among structures is demonstrated by recognizing part of the rubredoxin fold in the INSERT domain of the AKs. A search through the 612-structure subset is routinely feasible and requires about 2 days of $C P U$ time on a modern workstation. A reduced subset representing a lower level of sequence homology, or only those structures with a similar number of residues as the template, could be used to conserve CPU time.

The strength of the algorithm is certainly the exhaustive search over the entire angle space. Its use is only practical with contemporary computers, which make it possible to evaluate functions of computational complexity proportional to the product of the numbers of atoms involved at each grid point of angle space. Both the fast translation function and the topology similarity score are such functions. A potential disadvantage is the requirement for a relatively large amount of CPU time. As this is not a drawback in functionality, and computer technology seems to improve faster than the number of known unique protein topologies increases, this disadvantage will decrease in importance with time.

Compared to superposition methods that use difference vectors or distances the approach described here utilizes the available three-dimensional information without changing its representation. This change of representation is appealing because it enables a rotation and/or translation-invariant comparison of transformed entities. Once matching res- 
idues are found, the relative rotation and translation are calculated a posteriori. The crystallographic analogy to the comparison of difference vector sets is the comparison of Patterson functions. A program ${ }^{36}$ that generates the intramolecular difference vector sets from the coordinates and calculates a rotation and translation function in analogy to the crystallographic method of molecular replacement, does not perform as efficiently as the method described in this work: the signal-to-noise ratio of the "rotation function" is much lower than that observed for max${ }_{A}\left[Q_{A}(t)\right]$. A crystallographer's explanation is that it is harder to extract information from a Patterson than from a electron-density map. Algorithms working with sets of distances ${ }^{16}$ do not use the information about direction inherent to difference vectors. However, as the distances are "labeled" with the names of the contributing atoms, in principle no information is lost, as it is possible to reconstruct the coordinates from the distances by distance geometry methods.

In contrast to dynamic programming methods, the method described here can detect structures with similar fold but different order of SSEs (scoring functions FSS and RSS).

One of the main arguments against the sensitivity of a rigid-body superposition algorithm is the neglect of plasticity of a protein fold. The success of the algorithm presented here is due to the fact that although a polypeptide chain has many degrees of freedom, it usually packs tightly to form domains with a hydrophobic core. This physical requirement constrains the theoretically possible conformations to a finite number of motifs formed by SSEs. In the case of convergent evolution, the same structural motifs can be present in proteins that differ widely in their function and sequence. As demonstrated for colicin A, the resulting topological similarity is detectable by rigid-body superposition. This is even more the case for the proteins of the helical cytokine family, which are functionally similar and can be assumed to be related by divergent evolution.

Further possible applications of the method include the use of motifs or substructures (e.g. binding sites) as search templates. It is also ideally suited to searching with pseudo-atoms from a crystallographic "bones" structure, to identify which solved structures from the database may fit the electron density best. In this or in other cases, where the direction of the chain might be reversed, the RSS should be used instead of the TSS.

The algorithm might also be useful for the docking of molecules: in this case, coordinates extracted from the van der Waals surfaces of both molecules should be used as input coordinates. As the coordinates do not carry information about sequentiality, no filtering can be done.

Possible improvements include the use of optimized weights $w_{i j}$ and improved scoring functions. Savings in computer time by using a taboo search ${ }^{37}$ for the global maximum of the scoring function warrant investigation.

\section{ACKNOWLEDGMENTS}

The author thanks Drs. J.W. Coulton and W. Welte for critically reading the manuscript, and all scientists who deposit their structures in the Protein Data Bank.

\section{REFERENCES}

1. Richardson, J.S., Richardson, D.C., Thomas, K.A., Silverton, E.W., Davies, D.R. Similarity of three-dimensional structure between the immunoglobulin domain and the copper, zinc superoxide dismutase subunit. J. Mol. Biol. 102:221-235, 1976.

2. Holm, L., Sander, C. Globin fold in a bacterial toxin. Nature (London) 361:309, 1993.

3. Holm, L., Sander, C. Searching protein structure databases has come of age. Proteins 19:165-173, 1994.

4. Kabsch, W. A discussion of the solution for the best rotation to relate two sets of vectors. Acta Cryst. A34:827-828, 1978.

5. Hendrickson, W. Transformations to optimize the superposition of similar structures. Acta Cryst. A35:158-163, 1979.

6. McLachlan, A.D. Gene duplications in the structural evolution of chymotrypsin. J. Mol. Biol. 128:49-79, 1979.

7. Diamond, $R$. A note on the rotational superposition problem. Acta Cryst. A44:211-216, 1988.

8. Needleman, S.B., Wunsch, C.D. A general method applicable to the search for similarities in the amino acid sequence of two proteins. J. Mol. Biol. 48:443-453, 1970.

9. Taylor, W.R., Orengo, C.A. Protein structure alignment. J. Mol. Biol. 208:1-22, 1989.

10. Orengo, C.A., Brown, N.P., Taylor, W.R. Fast structure alignment for protein databank searching. Proteins 14: 139-167, 1992

11. Rose, J., Eisenmenger, F. A fast unbiased comparison of protein structures by means of the Needleman-Wunsch algorithm. J. Mol. Evol. 32:340-354, 1991.

12. Luo, Y., Lai, L., Xu, X., Tang, Y. Defining topological equivalences in proteins structures by means of a dynamic programming algorithm. Protein Eng. 6:373-376, 1993.

13. Sali, A., Blundell, T.L. Definition of general topological equivalence in protein structures. A procedure involving comparison of properties and relationships through simulated annealing and dynamic programming. J. Mol. Biol. 212:403 -428, 1990.

14. May, A.C.W., Johnson, M.S. Protein structure comparison using a combination of a genetic algorithm, dynamic programming and least-squares minimization. Protein Eng. 7:475-485, 1994

15. Mitchell, E.M., Artymiuk, P.J., Rice, D.W., Willet, P. Use of techniques derived from graph theory to compare secondary structure motifs in proteins. J. Mol. Biol. 212:151$166,1990$.

16. Holm, L., Sander, C. Protein structure comparison by alignment of distance matrices. J. Mol. Biol. 233:123-138, 1993.

17. Rossmann, M.G., Argos, P. Exploring structural homology of proteins. J. Mol. Biol. 105:75-95, 1976.

18. Schulz, G.E., Müller, C.W., Diederichs, K. Induced-fit movements in adenylate kinase. J. Mol. Biol. 213:627$630,1990$.

19. Lattman, E.E. Optimal sampling of the rotation function. Acta Cryst. B28:1065-1068, 1972.

20. Dayhoff, M.O. In "Atlas of Protein Sequence and Structure." Dayhoff, M.O., ed. Washington, DC: National Biomedical Research Foundation, 1979:353-358.

21. Diederichs, K., Boone, T., Karplus, P.A. Novel fold and putative receptor binding site of granulocyte-macrophage colony-stimulating factor. Science 254:1779-1782, 1991.

22. Parker, M.W., Pattus, F., Tucker, A.D., Tsernoglou, D. Structure of the membrane-pore-forming fragment of colicin A. Nature (London) 337:93-96, 1989.

23. Diederichs, K., Schulz, G.E. Three-dimensional structure of the complex between the mitochondrial matrix adeny- 
late kinase and its substrate AMP. Biochemistry 29:81388144,1990

24. Hobohm, U., Scharf, M., Schneider, R., Sander, C. Selection of representative protein data sets. Protein Sci. 1:409 $417,1992$.

25. Hobohm, U., Sander, C. Enlarged representative set of protein structures. Protein Sci. 3:522-524, 1994.

26. Bernstein, F.C., Koetzle, T.F., Williams, G.J.B., Meyer, E.F., Brice, M.D., Rodgers, J.R., Kennard, O., Shimanouchi, T., Tasumi, M. The Protein Data Bank: A computer based archival file for macromolecular structures. J. Mol Biol. 111:535-542, 1977.

27. Hill, C.P., Osslund, T.D., Eisenberg, D. The structure of granulocyte-colony-stimulating factor and its relationship to other growth factors. Proc. Natl. Acad. Sci. U.S.A. 90 5167-5171, 1993.

28. Ealick, S.E., Cook, W.J., Vijay-Kumar, S., Carson, M., Nagabhushan, T.L., Trotta, P.P., Bugg, C.E. Three-dimensional structure of recombinant human interferon- $\gamma$. Science 252:689-702, 1991

29. Robinson, R.C., Grey, L.M., Staunton, D., Vankelecom, H., Vernallis, A.B., Moreau, J.F., Stuart, D.I., Heath, J.K. Jones, E.Y. The crystal structure and biological function of leukemia inhibitory factor: implications for receptor binding. Cell 77:1101-1116, 1994.

30. Milburn, M.V., Hassell, A.M., Lambert, M.H., Jordan, S.R., Proudfoot, A.E., Graber, P., Wells,T.N. A novel dimer configuration revealed by the crystal structure at $2.4 \AA$ resolution of human interleukin-5. Nature (London) 363 172-176, 1993.

31. Holm, L., Sander, C. Structural alignment of globins, phycocyanins and colicin A. FEBS Lett. 315:301-306, 1993.

32. Jones, T.A., Zou, J.Y., Cowan, S.W., Kjeldgaard, M. Improved methods for building protein models in electron density maps and the location of errors in these models. Acta Cryst. A47:110-119, 1991.

33. Rozwarski, D.A., Gronenborn, A.M., Clore, G.M., Bazan, J.F., Bohm, A., Wlodawar, A., Hatada, M., Karplus, P.A Structure 2:159-173, 1994.

34. Glaser, P. Presecan, E., Delepierre, M., Surewicz, W.K. Mantsch, H.H., Bârzu, O., Gilles, A.-M. Zinc, a novel structural element found in the family of bacterial adenylate kinases. Biochemistry 31:3038-3043, 1992.

35. Perrier, V., Surewicz, W.K., Glaser, P., Martineau, L. Craescu, C.T., Fabian, H. Mantsch, H.H., Bârzu, O., Gilles, A.-M. Zinc chelation and structural stability of adenylate kinase from Bacillus subtilis. Biochemistry 33, 9960 9967, 1994.

36. Diederichs, K. SUPERIMPOSE-a program for the unambiguous structural superposition of spatially related molecules, including macromolecules. J. Appl. Cryst. 27:436, 1994.

37. Cvijovic, D., Jacek, K. Taboo search: An approach to the multiple minima problem. Science 267:664-666, 1995. 